Revue d'histoire de l'Amérique française

REVUE D.HISTOIRE DE L'AMÉRIQUE FRANÇAISE

\title{
"Point de fort pour la loi "? La justice civile dans la société acadienne de 1873 à 1899
}

\section{Jacques Paul Couturier}

Volume 45, numéro 2, automne 1991

URI : https://id.erudit.org/iderudit/304965ar

DOI : https://doi.org/10.7202/304965ar

Aller au sommaire du numéro

Éditeur(s)

Institut d'histoire de l'Amérique française

ISSN

0035-2357 (imprimé)

1492-1383 (numérique)

Découvrir la revue

Citer cet article

Couturier, J. P. (1991). « Point de fort pour la loi »? La justice civile dans la société acadienne de 1873 à 1899. Revue d'histoire de l'Amérique française, 45(2), 179-205. https://doi.org/10.7202/304965ar
Résumé de l'article

Cet article porte sur l'activité judiciaire acadienne dans les tribunaux civils néo-brunswickois du comté de Gloucester, de 1873 à 1899. Il tente de démontrer que les Acadiens, contrairement à l'image véhiculée par l'historiographie traditionnelle, ne boycottent pas systématiquement les mécanismes judiciaires officiels, du moins pas en matière civile. Ils les utilisent, moins que les anglophones, certes, mais de manière appréciable, compte tenu des domaines d'activité des tribunaux civils. Ce constat permet de s'interroger sur le degré de pluralisme régnant en matière juridique dans la société acadienne et sur son degré d'intégration à la société globale néo-brunswickoise. 


\title{
«POINT DE FORT POUR LA LOI»? LA JUSTICE CIVILE DANS LA SOCIÉTÉ ACADIENNE DE 1873 À $1899^{1}$
}

\author{
JACQUES PAUL COUTURIER \\ Departement d' histoire \\ Université du Nouveau-Brunswick
}

\section{RÉSUMÉ}

Cet article porte sur l'activité judiciaire acadienne dans les tribunaux civils néobrunswickois du comté de Gloucester, de 1873 à 1899. Il tente de démontrer que les Acadiens, contrairement à l'image véhiculée par l'historiographie traditionnelle, ne boycottent pas systématiquement les mécanismes judiciaires officiels, du moins pas en matière civile. Ils les utilisent, moins que les anglophones, certes, mais de manière appréciable, compte tenu des domaines d'activité des tribunaux civils. Ce constat permet de s'interroger sur le degré de pluralisme régnant en matière juridique dans la société acadienne et sur son degré d'intégration à la société globale néo-brunswickoise.

\section{ABSTRACT}

This article examines Acadian judicial activity in the civil courts of Gloucester County, New Brunswick, between 1873 and 1899. It argues that Acadians did not systematically boycott the judicial system in civil matters, contrary to the traditional interpretation. They did use the courts less frequently than anglophones. Yet this largely stems from the role civil courts played at the end of the century. This leads to questions about the degree of legal pluralism in Acadian society and the extent of Acadian integration in New Brunswick society as a whole.

1 Ce texte reprend une partie des conclusions de ma thèse de doctorat: La justice civile au Nouveau-Brunswick d la fin du 19e siècle: le cas du comté de Gloucester, entre 1873 et 1899 (thèse de Ph.D., histoire, Université de Montréal, 1990). Je tiens à remercier mon directeur de recherche, Pierre Trépanier, pour ses commentaires et son appui. Merci également à Maurice Basque, Philippe Garvie et Phyllis E. LeBlanc qui ont bien voulu commenter une première version du présent texte. Cette recherche a bénéficié du soutien financier du CRSH, du Fonds FCAR (programme Québec/Acadie) et du fonds de bourses de l'Université de Montréal. 
Depuis le début des années 1980, l'histoire du droit connaît au Canada un intérêt croissant. Pourtant, elle a encore peu de résonance dans l'historiographie générale. Cette situation découle principalement de la nature de la production: elle a longtemps été et demeure encore en partie axée sur des thèmes propres au droit et élaborée à partir de sources imprimées ${ }^{2}$. D'autres thèmes et d'autres approches, plus susceptibles de permettre à l'histoire du droit d'opérer une jonction avec l'histoire générale, notamment avec l'histoire sociale, sont encore trop peu explorés ${ }^{3}$. Il en est ainsi de la question de la culture juridique ${ }^{4}$. Ce thème présente un riche potentiel en contexte canadien, comme le démontrent notamment plusieurs travaux sur la réception du droit anglais au Québec durant la seconde moitié du 18e siècle et au $19 \mathrm{e}$ siècle ${ }^{5}$. Or le problème du changement de droit, de la réception d'un autre droit, n'est pas limité au seul cas québécois. Il se pose également pour d'autres groupes et à d'autres moments dans l'histoire canadienne: pour les Acadiens, par exemple, coupés des sources du droit français par le traité d'Utrecht en 1713, pour les immigrants autres que britanniques durant les périodes d'immigration massive des dernières décennies du $\mathrm{XIX}^{\mathrm{e}}$ siècle et du début du $\mathrm{XX}^{\mathrm{e}}$ siècle ou encore pour les communautés autochtones canadiennes. Bref la culture juridique canadienne n'est probablement pas uniforme; elle mérite d'être explorée plus avant, pour ce qu'elle peut révéler de l'expérience historique canadienne. La présente étude porte sur le cas acadien. Elle traite d'une dimension de la culture juridique: la place occupée par les mécanismes judiciaires officiels en matière civile - les tribunaux civils - dans la régulation juridique durant les dernières décennies du $\mathrm{XIX}^{\mathrm{e}}$ siècle. Elle vise à voir si, comme le notait l'avocat Charles Gagnon en 1886, les Acadiens n'ont «point de fort pour la loi» ${ }^{6}$.

2 Cette tendance est bien illustrée dans les deux premiers volumes de la collection Essays in the History of Canadian Law, publiés sous l'égide de la Osgoode Society et dirigés par David H. Flaherty: Essays in the History of Canadian Law - Vol. 1 (Toronto, University of Toronto Press, 1981), 428 p. et Essays in the History of Canadian Law - Vol. II (Toronto, The Osgoode Society, 1983), 593 p. Elle est toutefois nettement renversée dans le troisième volume. Voir Philip Girard et Jim Phillips, eds., Essays in the History of Canadian Law - Vol. III: Nova Scotia (Toronto, The Osgoode Society, 1990), $369 \mathrm{p}$.

3 À ce sujet, voir le plaidoyer d'Evelyn Kolish, «Some Aspects of Civil Litigation in Lower Canada, 1785-1825: Towards the Use of Court Records for Canadian Social History», Canadian Historical Review, 70,3 (1989): 337-365.

4 Soit «l'ensemble des opinions et des appréciations du public [...] sur le système juridique en vigueur». Voir André-Jean Arnaud, éd., Dictionnaire encyclopédique de théorie et de sociologie du droit (Paris, Librairie générale de Droit et de Jurisprudence, 1988), 89.

5 Notamment les travaux pionniers d'André Morel ou ceux plus récents d'Evelyn Kolish. Voir André Morel, «La réaction des Canadiens devant l'administration de la justice de 1764 à 1774: une forme de résistance passive», Revue du Barreau, 20 (février 1960): 55-63, et Evelyn Kolish, «Some Aspects...», 337-365.

6 Le Courrier des Provinces Maritimes (Bathurst, N.-B.), 24 mars 1886. 
De prime abord, le principal élément de toute enquête sur le droit dans la société acadienne semble être la question de la coupure avec les sources du droit français et l'introduction du droit anglais au début du XVIII ${ }^{e}$ siècle, à la suite de la conquête britannique ${ }^{7}$. Un autre élément est toutefois également de première importance: l'expérience juridique acadienne du siècle suivant, placée non plus sous le signe de l'acculturation juridique, mais plutôt sous celui d'un processus de rejudiciarisation ou même plus simplement de judiciarisation des rapports sociaux et économiques. La déportation, les années d'errance ou d'isolement qui s'ensuivent invitent à s'interroger sur le degré de pénétration de la culture juridique britannique ou officielle parmi la population acadienne. Celle-ci a peut-être encore à prendre la mesure du nouveau droit et du nouveau système judiciaire. Or la prise de contact ne semble s'établir que très lentement au fil du XIX ${ }^{\mathrm{e}}$ siècle, en raison notamment du peu de moyens de diffusion et de pénétration de la culture juridique officielle. Au Nouveau-Brunswick, par exemple, non seulement compte-t-on très peu d'intermédiaires - avocats, officiers de justice, magistrats, juges - pouvant faire office de pédagogues du droit avant le tournant du siècle ${ }^{8}$, mais les instruments de diffusion du droit anglais - lois, recueils de jurisprudence, traités, manuels - ne sont pas adaptés à la population francophone, étant tous de langue anglaise ${ }^{9}$. D'où l'intérêt de s'interroger sur le rythme et le degré de pénétration du droit et du système judiciaire officiel dans la société acadienne au XIX ${ }^{\mathrm{e}}$ siècle.

Tout le champ des rapports historiques entre droit et société dans la société acadienne a été très peu exploré ${ }^{10}$. On connaît peu de choses

7 Sur ce sujet, voir Thomas G. Barnes, «"The Daily Cry for Justice": the Judicial Failure of the Annapolis Royal Regime, 1713-1749», Girard et Phillips, eds., Essays in the History of Canadian Law - Vol. III, 10-41.

8 Jusqu'aux années 1870, les Acadiens sont très peu représentés au sein de l'appareil judiciaire de la province. Il n'y a pas d'avocat acadien avant 1870 , donc pas de juge acadien, et seulement quelques juges de paix. En 1900, on compte un juge acadien, huit avocats (excluant le juge Pierre-Armand Landry) et un certain nombre d'officiers de justice et de magistrats locaux. Malgré tout, le rapport avocat/population, par exemple, est encore de 1/9997 dans la communauté acadienne, contre 1/1138 dans l'ensemble de la province. Voir Couturier, La justice civile..., 93-122. Sur la période précédente, voir Marie-Claire Pitre, «Les juges de paix dans le comté de Gloucester, 1784-1867», Revue d' histoire de la Société historique Nicolas-Denys, 18,1 (janvier-avril 1990): 3-69.

9 Par exemple, il n'existe pas à la fin du XIX ${ }^{\mathrm{e}}$ siècle de manuel de juge de paix ou de guide juridique traitant en français de la common law. Il s'agit pourtant d'une voie privilégiée de diffusion de la culture juridique durant la période. On peut dresser un parallèle avec l'implantation du droit criminel anglais au Québec. Voir André Morel, «La réception du droit criminel anglais au Québec (1760-1892)», Revue juridique Thémis, 2-3 (1978): 449-542.

10 Les éléments qui suivent ont été énoncés dans un document intitulé «Préparation à l'inscription à la rédaction» déposé au département d'histoire de l'Université de Montréal en 1989. Marie-Claire Pitre y a eu accès et les a repris dans son texte «Les juges de paix...» et dans 
des attitudes des Acadiens face à la justice et moins encore de leurs pratiques en matière judiciaire. Tout au plus quelques éléments d'interprétation sont-ils proposés, notamment dans les travaux de Léon Thériault et de Lauraine Léger. Le plus souvent, la place du droit et des mécanismes judiciaires officiels y est dépeinte comme étant marginale, sinon inexistante ${ }^{11}$. On y suggère que les tribunaux mis en place par l'État demeurent largement inutilisés par la population acadienne, peut-être même encore au début du $\mathrm{XX}^{\mathrm{e}}$ siècle, principalement sous l'action de freins ethnolinguistiques ${ }^{12}$. Toute la problématique de la régulation juridique développée jusqu'à présent dans l'historiographie acadienne est toutefois axée sur la seule culture juridique informelle. Elle est appuyée presque exclusivement sur des sources extrajudiciaires, soit des pièces de correspondance ou des témoignages de contemporains. Par contre, l'univers de la justice officielle n'est pas directement exploré. C'est donc dire que le modèle développé dans l'historiographie ne rend pas compte de l'ensemble de la problématique de la régulation juridique dans la société acadienne.

Le présent article porte sur le volet négligé de la régulation juridique: la régulation juridique étatique. Il s'appuie sur un examen des archives judiciaires néo-brunswickoises dans un domaine précis d'intervention du droit, la solution des conflits relevant du droit privé, durant les dernières décennies du $\mathrm{XIX}^{\mathrm{e}}$ siècle ${ }^{13}$. Cet examen révèle une autre réalité de la régulation juridique. Il montre en effet que les Acadiens ne semblent pas éviter systématiquement et de manière généralisée les tribunaux civils néo-brunswickois. Ceux-ci s'en servent, certes de manière plus limitée que les anglophones, mais en grande partie parce que, moins présents dans le domaine du commerce et des affaires, moins bien dotés financièrement, ils ont moins besoin de tels instruments, qui sont surtout voués à la régulation des activités commerciales et financières. C'est donc dire que la culture juridique

un autre, «Les Acadiens et les juges de paix: étude des relations entre les Acadiens du Gloucester et le monde de la justice, 1784-1867», UNB Law Journal/Revue de droit UN.-B., 39 (1990): 171184 .

11 Voir Léon Thériault, «L'acadianisation de l'Église catholique en Acadie, 17631953», Jean Daigle, dir., Les Acadiens des Maritimes: études thématiques (Moncton, Centre d'études acadiennes, 1980), 319, et Lauraine Léger, Les sanctions populaires en Acadie - Région $d u$ comté de Kent (Montréal, Leméac, 1978), 186 p. Voir également David Bell, «A Perspective on Legal Pluralism in 19th-Century New Brunswick», UNB Law Journal/Revue de droit UN.B., 37 (1988): 89 .

12 Léger, Les sanctions..., 31-33.

13 C'est-à-dire le droit qui gouverne les rapports entre individus, entre personnes morales, et entre individus et personnes morales, à la différence du droit public, qui régit les rapports entre les individus et l'État ou l'ensemble du corps social et l'État lui-même. Ce droit recouvre notamment les trois grandes catégories étudiées ici: les contrats et dettes, la propriété et les délits civils. Voir Arnaud, Dictionnaire..., 118. 
acadienne est largement soumise à des déterminants socio-économiques à la fin du siècle. Ce constat ne conduit pas à nier la persistance d'une culture juridique informelle ou le rôle de mécanismes judiciaires non étatiques; il implique simplement que les mécanismes judiciaires étatiques ont aussi une place et jouent aussi un rôle dans la société acadienne. Bref, que le pluralisme juridique y prévaut durant les dernières décennies du XIX ${ }^{\mathrm{e}}$ siècle $^{14}$.

Notre examen de la régulation juridique dans la société acadienne est basé sur le cas du comté de Gloucester (N.-B.), durant la période 1873-1899. Ce choix est avant tout dicté par la disponibilité des sources. Les archives judiciaires de Gloucester sont parmi les mieux garnies des comtés à forte population acadienne de la province. Sont disponibles, pour la période, des archives de tribunaux des trois niveaux de compétence: la cour de circuit, la cour de comté et deux cours locales, celles de Beresford et de Saumarez ${ }^{15}$. Nous examinerons successivement trois dimensions du travail des tribunaux civils de Gloucester: le nombre de causes, la nature et le mode de règlement des instances, et finalement, le profil économique des plaideurs. Mais doivent d'abord être examinés les cadres de l'activité judiciaire.

\section{1 - LES CADRES DE L'ACTIVITÉ JUDICIAIRE}

Plusieurs éléments peuvent jouer sur l'activité judiciaire ${ }^{16}$. Trois semblent particulièrement significatifs dans le cas de Gloucester: la socio-économie locale, l'organisation de l'appareil judiciaire et la perception de la justice. Situé au nord-est du Nouveau-Brunswick, le

14 Sur le concept de pluralisme juridique, voir notamment Jean Carbonnier, Sociologie juridique (Paris, Presses universitaires de France, 1978), 204-218, et Jean-Guy Belley, «L'État et la régulation juridique des sociétés globales. Pour une problématique des pluralismes juridiques», Sociologie et sociétés, 18,1 (avril 1986): 11-32. Pour une application du concept en contexte néo-brunswickois, voir Bell, «A Perspective...», 86-93.

15 Ces documents sont tous conservés aux Archives provinciales du NouveauBrunswick [APNB]. Pour la cour de circuit, les références sont: Supreme Court Records: Original Jurisdiction, RG 5, RS 42, séries «de» [declarations] et «Z» [unbroken bundles], 18731899; Supreme Court Records: Judgment Rolls, RG 5, RS 51, B/5-7, Indices, 1873-1899. Pour la cour de comté: Gloucester County Court Records, RG 6, RS 432, A, Case files, 1873-1899 (largement incomplets entre 1890 et 1899); B/1, Plaintiffs' Book, 1873-1899; B/2, Defendants' Book, 1873-1899 et B/3, County Court Register, 1879-1899. Pour les cours civiles paroissiales: Gloucester Parish Civil Court Records: Parish Civil Court Record Books, RG 8, RS 495, A/2a, Beresford, 1890-1896 et Justice of the Peace Records for Gloucester County: Justices Court Record Books, RG 8, RS 480, E/2-a, Saumarez, 1887-1898.

16 Voir Stephen Daniels, «Ladders and Bushes: the Problems of Caseloads and Studying Court Activities Over Time», American Bar Foundation Research Journal, 4 (1984): 789-791, et Frank Munger, «Law, Change, and Litigation: a Critical Examination of an Empirical Research Tradition», Law and Society Review, 22,1 (1988): 58-62. 
comté de Gloucester est l'un des plus acadiens de la province au tournant du siècle. En $1871,67,4 \%$ de ses 18810 habitants sont d'origine française. Ce pourcentage est en progression constante durant toute la période, atteignant $80,9 \%$ en 1901 , sur une population totale de 27936 personnes ${ }^{17}$. Presque toute l'activité économique y est concentrée dans le secteur primaire ${ }^{18}$. Cependant, l'agriculture et la pêche, faiblement ou mal commercialisées, sont des activités peu profitables pour les individus qui les pratiquent. Elles sont souvent exercées pour des fins de subsistance, d'où l'ampleur du phénomène des occupations multiples ${ }^{19}$. Certains secteurs sont de plus dominés par quelques grands intervenants, que ce soit la pêche par les compagnies Robin et Fruing ou l'exploitation forestière par Kennedy F. Burns. D'ailleurs les effectifs du groupe des gens d'affaires - marchands, commerçants, négociants, agents, hôteliers - sont peu nombreux, ne regroupant que $1,1 \%$ de la main-d'œuvre en $1881^{20}$. Or seulement un sixième des gens d'affaires du comté sont Acadiens la même année. Dix ans plus tard, les Acadiens ne constituent encore qu'un peu plus du tiers des effectifs de la communauté marchande ${ }^{21}$. Ce sous-développement économique a pour corollaire un sous-développement social, caractérisé notamment par un fort degré d'analphabétisme. Près de la moitié des habitants du comté âgés de plus de 20 ans déclarent ne savoir ni lire ni écrire lors du recensement de $1891^{22}$. Ces différents éléments peuvent tous avoir une incidence négative sur le recours judiciaire.

Trois tribunaux se partageaient la plus grande partie de la charge de travail en matière de litiges civils dans le comté durant les dernières décennies du XIX ${ }^{\mathrm{e}}$ siècle: la cour de circuit, la cour de comté et les cours civiles paroissiales. C'est la cour de circuit qui est chargée des cas les plus importants ${ }^{23}$. Sa compétence pécuniaire est illimitée. Elle

17 Canada, ministère de l'Agriculture, Recensement du Canada, 1871, vol. I, tabl. III et Ibid., 1901, vol. I, tabl. XI.

18 Voir Donald J. Savoie et Maurice Beaudin, La lutte pour le développement - Le cas du Nord-Est, Québec (Presses de l'Université du Québec et Institut canadien de recherche sur le développement régional, 1988), 21-43.

19 À titre d'exemple, voir l'analyse de Nicolas Landry sur les fermiers-pêcheurs: Aspects socio-économiques des régions côtières de la Péninsule acadienne (NouveauBrunswick), 1850-1900 (thèse de M.A., histoire, Université de Moncton, 1982), 31-83.

20 Recensement du Canada, 1881, vol. II, tabl. XIV.

21 Données basées sur une compilation sommaire des listes nominatives des recensements de 1881 et 1891 . Des résultats similaires ont été obtenus à partir des données des annuaires de crédit de la firme Dun, Wiman \& Co. Voir The Mercantile Agency Reference Book (and Key) for the Dominion of Canada (Montréal, Dun, Wiman \& Co., juillet 1882) et Ibid., juillet 1890 .

22 Recensement du Canada, 1891, vol. II, tabl. XIII.

23 Consolidated Statutes of New Brunswick, 1877, ch. 22. Sur l'organisation judiciaire néo-brunswickoise au XIX' ${ }^{\circ}$ siècle, voir J. A. Clarence Smith et Jean Kerby, Le droit privé au Canada - Études comparatives (Ottawa, Presses de l'Université d'Ottawa, 1987), 100-101, 105106, 108-109, 113, 116-117. Voir aussi Couturier, La justice civile..., ch. II. 
seule peut connaître les actions en matière de contestation de titres de propriété. Elle siège d'abord une fois, puis deux fois par année dans le comté, sous la responsabilité d'un juge de la Cour suprême. Quant à la cour de comté, elle est habilitée à entendre les actions sur dettes, engagements ou promesses, si la dette ou les dommages-intérêts réclamés n'excèdent pas $200 \$$. Elle peut aussi entendre les actions en responsabilité délictuelle, lorsque les dommages-intérêts demandés ne dépassent pas $100 \$$, et les actions sur cautionnement en garantie d'exécution. Ces plafonds pécuniaires seront doublés en $1882^{24}$. La cour de comté de Gloucester siège trois fois par année, en mars, juillet et novembre. Au bas de la hiérarchie judiciaire, les cours civiles paroissiales, instituées en 1876, ont principalement la responsabilité des petites créances ${ }^{25}$. Elles jouissent à l'origine d'une compétence limitée à $40 \$$ en matière de dettes et à $16 \$$ en matière de délits civils. Ces limites pécuniaires seront haussées à $80 \$$ et à $32 \$$ en $1882^{26}$. À la différence des deux autres cours, les cours civiles paroissiales sont sous la responsabilité de magistrats non professionnels. Par ailleurs, peu importe le tribunal, toutes les procédures doivent officiellement être menées en anglais, une situation qui prévaudra jusqu'en $1970^{27}$. Cette prescription semble être respectée devant la Cour suprême et la cour de comté, mais ne l'est peut-être pas totalement dans les tribunaux inférieurs. Certaines parties du procès s'y déroulent parfois en français, sans l'intermédiaire d'un interprète, lorsque le magistrat est en mesure de comprendre la langue ${ }^{28}$. Mais, règle générale, la justice s'exprime en anglais dans une région largement francophone.

Sur les plans de la représentativité de l'appareil judiciaire et de la perception de la justice, les conditions apparaissent également peu favorables aux activités litigieuses. Sauf dans l'incarnation inférieure de l'appareil judiciaire, les cours civiles paroissiales, le personnel judich. 9.

24 Statuts du Nouveau-Brunswick, 30 Vict. (1867), ch. 10, art. 8 et Ibid., 45 Vict. (1882),

25 Ibid., 39 Vict. (1876), ch. 5. Les autres tribunaux inférieurs dans Gloucester étant les cours de magistrats rétribués, établies à Bathurst et à Caraquet, et les cours des juges de paix, présentes partout où réside un juge de paix, soit habituellement dans chaque paroisse.

26 Ibid., 45 Vict. (1882), ch. 11, art. 1-2.

27 Michel Bastarache, «Droits linguistiques et culturels des Acadiens de 1713 à nos jours», Les Acadiens..., 406-407.

28 Ce qui donne parfois lieu à des situations ambiguës. À preuve cette description de l'audition d'une cause impliquant un Irlandais et un Acadien par le juge de paix Jonathan Doran de Shippagan en 1879: «I administered the oath in English. I usually talk to him in French. [...] I can't say that the prisoner can understand English. He may understand a few words. [...] The evidence in this case was given in French. I put it in my book. I won't swear that I can translate English unto French correctly.» APNB, RG 6, RS 432, A, boîte 1809-1886, dossier Rex c. Mallet, 1880. Voir Couturier, La justice civile..., 90-91, et Bastarache, «Droits linguistiques...», 407. 
ciaire du comté est presque totalement formé d'Anglo-Brunswickois. Juges, greffiers, avocats sont anglophones, à la seule exception des shérifs Laman R. Doucet (1881-1893) et Onésime Blanchard (à partir de 1893), et de l'avocat Narcisse A. Landry (à partir de 1880). La pénurie d'avocat est particulièrement prononcée: les Acadiens peuvent compter sur un seul avocat résidant pour 22599 personnes en 1900, tandis que le rapport avocat population/totale est de 1/5587 à l'échelle du comtée ${ }^{29}$. Cette sous-représentation dans le domaine judiciaire, associée à l'unilinguisme des procédures supérieures, alimente d'ailleurs la perception négative des tribunaux et du recours judiciaire qui semble prévaloir dans la société acadienne durant les dernières décennies du $\mathrm{XIX}^{\mathrm{e}}$ siècle. Jugé non représentatif, l'appareil judiciaire est perçu comme opérant au désavantage de la population acadienne ${ }^{30}$. Or cette perception vient en renforcer une autre présente dans plusieurs milieux au Canada au XIX ${ }^{\mathrm{e}}$ siècle: le caractère nuisible et néfaste des litiges civils $^{31}$. Tout comme sur le plan de la socio-économie, la perception des tribunaux semble donc peu favorable au développement des activités litigieuses dans la communauté acadienne de Gloucester. Les pressions à l'évitement sont grandes, particulièrement au niveau des cours de circuit et de comté. C'est là que les conditions locales ont probablement le plus d'impact.

\section{2 - LE NIVEAU D'ACTIVITÉ JUDICIAIRE}

«Il est consolant de voir, note le Courrier des Provinces Maritimes en 1899, que dans un comté aussi français que Gloucester il n'y [a] pas un seul procès civil sur le dossier où un français [est] mis en cause. $\rangle^{32}$ Toutes les sessions de la cour de comté de Gloucester ne sont pas comme celle décrite par le journal de Bathurst. Néanmoins, l'activité judiciaire acadienne dans les tribunaux civils locaux apparaît effectivement peu imposante entre 1873 et $1899^{33}$. Elle est généralement en deçà de la présence acadienne parmi la population du

29 Données compilées à partir du Recensement du Canada, 1901, vol. I, tabl. VII et XI et du McMillan's Almanac for 1900 (St.John, J. \& A. McMillan, 1900).

30 Voir Couturier, La justice civile..., 123-151.

31 À titre d'illustration, voir J. E. Hansford, The Business Guide; or, Safe Methods of Business (Toronto, J. L. Nichols, 1892), 12, un ouvrage d'initiation aux affaires destiné à un large public. Ce thème rejoint celui de la suspicion populaire à l'endroit des avocats, assez répandue à l'époque. Voir Greg Marquis, "Anti-Lawyer Sentiment in Mid-Victorian New Brunswick», UNB Law Journal/Revue de droit UN.-B., 36 (1987): 163-174.

32 Le Courrier des Provinces Maritimes, 21 mars 1899.

33 Par activité judiciaire, nous entendons le nombre d'actions introduites plutôt que le nombre d'actions contestées ou le nombre de procès. Cette approche semble préférable, car elle permet de prendre en charge la contribution pré-décisionnelle des tribunaux dans le règlement 
TABLEAU 1

Nombre d'actions acadiennes, anglo-brunswickoises et totales dans les tribunaux civils de Gloucester, de 1873 à 1899

A. Tribunaux supérieurs

\begin{tabular}{|c|c|c|c|c|c|c|}
\hline \multirow[b]{2}{*}{ Année } & \multicolumn{3}{|c|}{ Circuit } & \multicolumn{3}{|c|}{ Comté } \\
\hline & $\mathbf{A}$ & B & $\mathbf{T}$ & $\mathbf{A}$ & B & $\mathbf{T}$ \\
\hline & Nbre (\%) & Nbre (\%) & & Nbre (\%) & Nbre (\%) & \\
\hline 1873 & 1 & 8 & 10 & 8 & 32 & 53 \\
\hline 1874 & 1 & 7 & 13 & 5 & 30 & 53 \\
\hline 1875 & 0 & 10 & 14 & 4 & 23 & 53 \\
\hline 1876 & 0 & 8 & 18 & 6 & 32 & 63 \\
\hline 1877 & 0 & 2 & 6 & 5 & 28 & 69 \\
\hline 1878 & 2 & 3 & 8 & 7 & 23 & 47 \\
\hline 1879 & 0 & 2 & 4 & 4 & 10 & 34 \\
\hline 1880 & 0 & 3 & 5 & 2 & 23 & 29 \\
\hline 1881 & 0 & 3 & 7 & 2 & 25 & 37 \\
\hline s.t. & $4(4,7)$ & $46(54,1)$ & 85 & $43(9,8)$ & $226(51,6)$ & 438 \\
\hline 1882 & 2 & 0 & 5 & 7 & 8 & 21 \\
\hline 1883 & 2 & 1 & 3 & 8 & 15 & 30 \\
\hline 1884 & 0 & 2 & 2 & 1 & 6 & 20 \\
\hline 1885 & 0 & 2 & 3 & 2 & 20 & 37 \\
\hline 1886 & 2 & 1 & 5 & 2 & 10 & 23 \\
\hline 1887 & 0 & 3 & 6 & 3 & 7 & 25 \\
\hline 1888 & 1 & 1 & 3 & 1 & 12 & 18 \\
\hline 1889 & 3 & 2 & 6 & 2 & 3 & 10 \\
\hline 1890 & 1 & 1 & 2 & 7 & 2 & 19 \\
\hline s.t. & $11(31,4)$ & $13(37,1)$ & 35 & $33(16,3)$ & $83(40,9)$ & 203 \\
\hline 1891 & 0 & 3 & 5 & 5 & 3 & 18 \\
\hline 1892 & 0 & 1 & 6 & 8 & 5 & 22 \\
\hline 1893 & 2 & 3 & 5 & 4 & 2 & 11 \\
\hline 1894 & 3 & 4 & 9 & 3 & 1 & 18 \\
\hline 1895 & 1 & 0 & 3 & 0 & 1 & 10 \\
\hline 1896 & 1 & 1 & 4 & 4 & 4 & 19 \\
\hline 1897 & 2 & 1 & 3 & 1 & 7 & 14 \\
\hline 1898 & 2 & 0 & 4 & 7 & 5 & 21 \\
\hline 1899 & 2 & 1 & 3 & 8 & 9 & 23 \\
\hline s.t. & $13(31,0)$ & $14(33,3)$ & 42 & $40(25,6)$ & $37(23,7)$ & 156 \\
\hline Total & $28(17,3)$ & $73(45,1)$ & 162 & $116(14,6)$ & $346(43,4)$ & 797 \\
\hline
\end{tabular}

Source: voir note 14 .

A: Acadiens, B: Anglo-Brunswickois de Gloucester, T: Total 


\section{TABLEAU 1 (SUITE)}

B. Inférieurs: Beresford et Saumarez

\begin{tabular}{|c|c|c|c|c|c|c|}
\hline \multirow[b]{2}{*}{ Année } & \multicolumn{3}{|c|}{ Beresford } & \multicolumn{3}{|c|}{ Saumarez } \\
\hline & $\mathbf{A}$ & B & $\mathbf{T}$ & $\mathbf{A}$ & B & $\mathbf{T}$ \\
\hline & Nbre (\%) & Nbre (\%) & & Nbre (\%) & Nbre (\%) & \\
\hline 1887 & & & & $5 *$ & $45^{*}$ & $54 *$ \\
\hline 1888 & & & & 2 & 32 & 36 \\
\hline 1889 & & & & 5 & 8 & 20 \\
\hline 1890 & $35^{*}$ & $4 *$ & $41 *$ & 2 & 38 & 43 \\
\hline 1891 & 72 & 24 & 97 & 2 & 17 & 34 \\
\hline 1892 & 74 & 10 & 92 & 9 & 34 & 44 \\
\hline 1893 & 61 & 13 & 75 & 13 & 51 & 65 \\
\hline 1894 & 62 & 3 & 66 & 4 & 59 & 63 \\
\hline 1895 & 71 & 6 & 78 & 5 & 18 & 23 \\
\hline 1896 & $47 *$ & $6 *$ & $58 *$ & 1 & 18 & 20 \\
\hline 1897 & & & & 5 & 17 & 22 \\
\hline 1898 & & & & $11^{*}$ & $31 *$ & $42 *$ \\
\hline Total 4 & $422(83,2)$ & $66(13,0)$ & 507 & $64(13,7)$ & $368(79,0)$ & 466 \\
\hline
\end{tabular}

Source: voir note 14 .

A: Acadiens, B: Anglo-Brunswickois de Gloucester, T: Total

* Années incomplètes

comté. C'est le cas en cour de circuit (voir tableau 1). Seulement 28 des 162 actions présentées devant le tribunal entre 1873 et 1899 le sont par des Acadiens ou comptent un Acadien parmi le groupe des demandeurs ${ }^{34}$. Ce chiffre constitue une sous-évaluation du nombre de causes, compte tenu de la source utilisée, les déclarations ${ }^{35}$, mais rien n'indique qu'il ne peut pas refléter adéquatement l'évolution dans le

des litiges. Voir Couturier, La justice civile..., 157-182. Voir également Richard Lempert, «More Tales of Two Courts: Exploring the «Dispute Settlement Function» of Trial Courts», Law and Society Review, 13,1 (1978): 97-100.

34 C'est-à-dire une personne d'origine française résidant dans Gloucester. Une action intentée par un marchand acadien d'un autre comté, par exemple, n'est donc pas comptabilisée dans le total de la contribution acadienne, alors que l'est celle d'un marchand d'origine québécoise demeurant dans Gloucester. Notre analyse ne met par ailleurs pas l'accent sur la variable sexuelle. Peu d'Acadiennes, peu de femmes, en fait, interviennent devant les tribunaux civils. Elles sont présentes dans $3,0 \%$ à $4,0 \%$ des actions dans les trois tribunaux. Voir Couturier, La justice civile..., 321-325.

35 Seules les déclarations permettent en effet d'évaluer le degré d'activité judiciaire à l'échelle d'un comté en cour de circuit, étant donné que le registre des causes du tribunal ne contient pas d'indications sur le lieu d'audience. Ce choix entraîne une sous-estimation du 
temps de la charge de travail du tribunal. Or, au fil des ans, l'activité judiciaire acadienne fluctue peu. Ainsi, l'écart entre le plus grand et le plus petit nombre de causes d'une année à l'autre n'est que de trois. Cependant, elle montre des signes de progression entre le début et la fin de la période. Alors qu'il n'y a que quatre causes acadiennes durant les neuf premières années (1873-1881), on en compte 11 durant les neuf suivantes (1882-1890) et 13 durant les neuf dernières (18911899). Ce mouvement à la hausse est contraire à l'évolution du nombre total de causes, qui passe de 85 à 35 puis à 42 durant les trois périodes examinées. C'est donc dire que les autres plaideurs sont de moins en moins actifs. Deux facteurs peuvent notamment expliquer l'évolution du niveau général d'activité: un déplacement de clientèle, suite à la création d'un nouveau tribunal à l'échelon inférieur en 1876, les cours civiles paroissiales ${ }^{36}$, et une diminution de l'activité des principaux utilisateurs de l'appareil judiciaire civil, les marchands et les entreprises commerciales ${ }^{37}$. Cette diminution du nombre total de causes, associée au léger accroissement de l'activité judiciaire acadienne, fait que celle-ci occupe progressivement une plus grande partie de l'activité judiciaire de la cour de circuit. Au cours de la période 1873-1881, 4,7\% des causes impliquent au moins un demandeur acadien. Ce pourcentage grimpe à environ $31 \%$ durant les deuxième et troisième périodes. La tendance de l'activité judiciaire acadienne en cour de circuit apparaît donc assez nettement: elle n'augmente que légèrement, mais représente toutefois peu à peu une plus grande part de l'activité judiciaire totale.

Dans l'autre tribunal ayant ressort sur tout Gloucester, la cour de comté, les Acadiens présentent 116 actions entre 1873 et 1899 , soit $14,6 \%$ des 797 actions introduites. Cette présence varie de zéro à huit causes par année. Par contre, sur l'ensemble de la période, l'activité judiciaire acadienne ne connaît ni de véritable avancée, ni de véritable recul. Quarante-trois actions sont inscrites par des Acadiens entre 1873 et 1881,33 entre 1882 et 1890 , ainsi que 40 entre 1891 et 1899. Tout comme en cour de circuit et pour les mêmes raisons, l'activité judiciaire générale se distingue de celle des Acadiens. Elle ne présente pas les mêmes éléments de stabilité, mais est plutôt nettement à la

nombre total d'actions intentées, étant donné que certaines ont pu ne pas se rendre à l'étape de la déclaration, se terminant par un jugement par défaut à partir d'un bref portant mention spéciale ou par un règlement hors cours. Ce problème ne se pose pas en cour de comté et dans les deux cours civiles paroissiales étudiées, chacune possédant son propre registre.

36 Couturier, La justice civile..., 247-252.

37 Ce dernier élément est probablement tributaire de modifications à l'intérieur du système commercial et financier canadien, en particulier l'extension du réseau bancaire et de meilleures possibilités de renseignement sur le crédit. Voir Ibid., 337-340. 
baisse. Ainsi, entre 1873 et 1881, la moyenne de causes par année est de 48,7; pour les années suivantes, elle s'établit plutôt à 19,9, soit une chute de $59,1 \%$. Par conséquent, l'activité judiciaire acadienne devient donc plus visible au fil de la période. Elle passe de $9,8 \%$ à $16,3 \%$, puis à $25,6 \%$ de toutes les causes portées au registre du tribunal durant les trois périodes examinées. Ces gains dépendent toutefois uniquement de la diminution de l'activité judiciaire des autres plaideurs. À la différence de la cour de circuit, en effet, l'activité judiciaire acadienne varie peu durant toute la période.

De prime abord, la nature même des cours civiles paroissiales et la présence de deux commissaires acadiens, Olivier Robichaud dans Saumarez et Perry J. Comeau dans Beresford, portent à penser que l'activité judiciaire acadienne pourrait être nettement supérieure à l'échelon local. Cette hypothèse se vérifie dans Beresford. Sur un total de 507 causes entendues entre 1890 et 1896, 422 sont initiées par des Acadiens, ce qui représente $83,2 \%$ du total. La contribution acadienne y varie d'une année à l'autre, oscillant entre $74,2 \%$ et $93,9 \%$ des causes entendues. Ce qui est vrai pour le tribunal de Beresford ne l'est toutefois pas pour celui de Saumarez. Dans le tribunal du commissaire Robichaud, les Acadiens ne revendiquent que la part congrue de l'activité judiciaire. Seulement 64 des 466 causes inscrites au registre comptent au moins un demandeur acadien entre 1887 et 1898 , soit $13,7 \%$ du total. Ce pourcentage change année après année, s'établissant aux extrêmes à $4,7 \%$ en 1890 et à $26,2 \%$ en 1898 , sans toutefois présenter de tendance particulière sur l'ensemble de la période. Or la paroisse compte proportionnellement plus d'Acadiens que celle de Beresford en $1891,94,1 \%$ contre $87,5 \%{ }^{38}$. Ces deux cours civiles paroissiales présentent donc un portrait complètement différent de l'activité judiciaire acadienne. Ceci ne signifie toutefois pas que les habitants du Saumarez boycottent les tribunaux locaux, la paroisse comptant plus d'une cour locale. Ils semblent plutôt simplement bouder celui de Robichaud. Ainsi, à l'échelle locale, l'activité judiciaire apparaît fortement liée aux conditions propres à chaque cour ${ }^{39}$.

Confrontée à l'activité judiciaire totale, l'activité judiciaire acadienne apparaît donc dans la plupart des cas fort limitée. Dans un tribunal, la cour de circuit, elle est en progression en termes absolus, mais n'atteint jamais une position prédominante, même dans la

38 Recensement du Canada, 1891, vol. I, tabl. III.

39 Dans ce cas-ci, en particulier, à la personne et au rôle du commissaire. Robichaud est peut-être victime de son apparente association avec la société John \& Richard Young. Celle-ci est en effet responsable d'environ la moitié des actions entendues par le magistrat entre 1887 et 1895. Voir Couturier, La justice civile..., 264, 427-430. 
dernière décennie du siècle. Dans un deuxième, la cour de comté, elle demeure sensiblement la même, mais profite toutefois de la diminution de l'activité judiciaire globale pour occuper proportionnellement une plus grande part du rôle. Dans les deux derniers, des cours civiles paroissiales, elle varie annuellement, sans présenter de tendance au fil des ans. Elle domine largement dans Beresford, mais est nettement au second plan dans Saumarez. De plus, dans les deux tribunaux supérieurs, l'examen du taux de litiges pour mille habitants - une mesure statistique qui permet de déborder le cadre de la seule comparaison intrajudiciaire ${ }^{40}$ - met en relief la relative stabilité de l'activité judiciaire sur l'ensemble de la période 1873-1899. Ainsi, en cour de circuit, le taux moyen de causes pour mille habitants dans la communauté acadienne est de 0,03 entre 1873 et 1881 , de 0,07 entre 1882 et 1890 et toujours de 0,07 entre 1891 et 1899 . Il y a donc une faible hausse entre la première et la deuxième période, puis coïncidence parfaite entre la deuxième et la dernière. On retrouve les mêmes éléments de stabilité en cour de comté. Le taux moyen de causes pour mille habitants est successivement de 0,34, 0,22 et 0,22 dans les trois périodes étudiées. En ce qui a trait aux deux tribunaux supérieurs, il est donc clair que le niveau de recours judiciaire acadien ne progresse pas de manière significative au cours des dernières décennies du $\mathrm{XIX}^{\mathrm{e}}$ siècle. Tout au plus peut-on déceler de faibles indices de changement entre la première et la deuxième période, sans plus. C'est donc l'augmentation de la population acadienne qui rend le mieux compte des changements du niveau d'activité judiciaire acadienne entre 1873 et 1899 .

Il faut toutefois se garder de conclure immédiatement que les Acadiens de Gloucester fréquentent nettement moins les tribunaux civils que les autres groupes du comté. Une partie des causes non acadiennes sont en effet présentées par des intervenants de l'extérieur du comté, des marchands de Montréal, de Québec ou de Saint-Jean, par exemple; une autre l'est par des personnes morales, soit des compagnies, des administrations municipales ou des conseils scolaires. $\mathrm{Si}$ l'on tient compte de la seule activité judiciaire générée par des individus résidant dans Gloucester, la contribution acadienne apparaît nettement moins marginale, même si elle demeure toujours secondaire (voir plus haut, tableau I) ${ }^{41}$. Ainsi 73 actions sont inscrites par des Anglo-Brunswickois résidant dans Gloucester en cour de circuit entre

40 Lempert, «More Tales...», 93-97.

41 Le lieu de résidence a été établi au moyen des archives judiciaires et des recensements nominatifs. Un certain nombre de causes n'ont pu être identifiées: elles ont donc été classées dans une catégorie «indéterminée». 
1873 et 1899 , contre 28 par des Acadiens. Cette prépondérance est établie durant la première période (1873-1881): 46 actions sont attribuables au premier groupe, seulement quatre au second. Dans les deux autres périodes, toutefois, Anglo-Brunswickois et Acadiens de Gloucester présentent sensiblement le même nombre de causes: 13 contre 11 dans les années $1882-1890$ et 14 contre 13 dans les années 1891-1899 ${ }^{42}$. L'écart est également réduit en cour de comté. Trois cent quarante-six causes sont présentées par des plaideurs anglophones locaux, contre 116 par des Acadiens. Par contre, le point de rencontre des deux groupes y est plus tardif que dans le premier tribunal. Il ne se situe pas avant les années 1890 . Trente-sept actions sont alors introduites par des anglophones contre 40 par des francophones. C'est encore dans la première période que l'écart entre les deux groupes est le plus prononcé, la quarantaine d'actions acadiennes étant nettement supplantées par les 226 actions anglo-brunswickoises ${ }^{43}$. La situation demeure cependant inchangée dans les tribunaux inférieurs. Presque toutes les actions y sont intentées, d'une manière ou d'une autre, par des gens habitant Gloucester. Ces quelques données sur l'activité judiciaire locale ramènent donc à des dimensions plus modestes l'écart entre les contributions acadienne et anglo-brunswickoise. Elles laissent déjà entrevoir que les Acadiens du comté ont moins recours à l'appareil judiciaire civil que les autres habitants du comté ${ }^{44}$, mais qu'ils ne les évitent pas pour autant systématiquement.

Les Acadiens sont plus présents dans les tribunaux civils de Gloucester à titre de défendeur (voir tableau 2) ${ }^{45}$. Ainsi, environ le tiers des causes sont défendues par des Acadiens en cour de circuit. Cette proportion progresse entre la première et la deuxième période, puis se stabilise. Elle est de $29,4 \%$ dans la période $1873-1881$, de $48,6 \%$ dans la période $1882-1890$ et de $45,2 \%$ dans la période 1891 1899. Par tranche chronologique, la présence acadienne est quelque peu différente en cour de comté. Elle est proportionnellement plus forte durant la première et la dernière période que durant la période intermédiaire. Cependant, elle est identique à celle enregistrée en cour

42 Même en ajoutant les causes «indéterminées» à celles identifiées comme provenant de plaideurs anglophones locaux, l'écart demeure peu significatif. On compte alors 15 causes contre 11 dans la deuxième période et 16 contre 13 dans la troisième.

43 Dans les deux cas, la position des anglophones est renforcée par l'ajout des actions dites «indéterminées». Elle devient notamment prépondérante dans la dernière période, s'établissant à 57 pour les premiers et à 40 pour les seconds.

44 Dans une étude sur les tribunaux civils du Bas-Canada, Evelyn Kolish a constaté un phénomène similaire: la sous-représentation des Canadiens parmi les plaideurs. Voir Kolish, «Some Aspects...», 348.

45 Ce classement comprend tous les défendeurs francophones, qu'ils soient ou non résidants du comté. 


\section{TABLEAU 2}

Nombre d'actions défendues par des Acadiens et interaction ethnolinguistique dans les tribunaux civils de Gloucester, de 1873 à 1899.

\begin{tabular}{lccc}
\hline & $\begin{array}{c}\text { Défendeurs } \\
\text { acadiens (a) }\end{array}$ & $\begin{array}{c}\text { Couple } \\
\text { homogène (b) }\end{array}$ & $\begin{array}{c}\text { Couple } \\
\text { Mixte (c) }\end{array}$ \\
\hline Tribunal/Période & Nbre (\%) & Nbre (\%) & Nbre (\%) \\
\hline Circuit & & & \\
$1873-81$ & $25(29,4)$ & 2 & 9 \\
$1882-90$ & $17(48,6)$ & 8 & 3 \\
$1891-99$ & $19(45,2)$ & 12 & 5 \\
Total & $\mathbf{6 1}(\mathbf{3 7 , 7})$ & $\mathbf{2 2}(\mathbf{7 8 , 6 )}$ & $\mathbf{1 7}(\mathbf{2 3 , 3 )}$ \\
Comté & & & \\
$1873-81$ & $180(41,1)$ & 24 & 96 \\
$1882-90$ & $65(32,0)$ & 15 & 25 \\
$1891-99$ & $62(39,7)$ & $\mathbf{5 6}(\mathbf{4 8 , 3 )}$ & $\mathbf{1 3 3}(\mathbf{3 8 , 4 )}$ \\
Total & $\mathbf{3 0 7}(\mathbf{3 8 , 5 )}$ & & \\
Inférieurs & & $385(91,2)$ & $66(100)$ \\
Beresford & $462(91,1)$ & $55(85,9)$ & $316(85,9)$ \\
Saumarez & $403(86,5)$ & & \\
\hline
\end{tabular}

Source: voir note 14.

A: Causes défendues par des Acadiens / \% du total des causes.

B: Causes dont le demandeur et le défendeur sont Acadiens / \% des causes acadiennes.

C: Causes dont le défendeur est Acadien et le demandeur un anglophone du comté / \% des causes anglophones.

de circuit sur l'ensemble de la période 1873-1899. Au total, 307 causes, soit $38,5 \%$ des causes, sont défendues par des Acadiens. Une situation différente prévaut dans les tribunaux inférieurs de Beresford et de Saumarez. Sur un total de 973 causes dans les deux cours, 88,9\% ont au moins un défendeur acadien. Cette proportion varie légèrement entre les deux tribunaux. Elle est de $91,1 \%$ dans Beresford et de $86,5 \%$ dans Saumarez. Par rapport aux demandeurs, la présence acadienne est donc plus forte parmi le groupe des défendeurs, à tous les niveaux de l'appareil judiciaire. Mais même dans ce dernier groupe, l'élément acadien reste la plupart du temps sous-représenté, compte tenu de son poids démographique. C'est donc dire que, dans une bonne mesure, l'appareil judiciaire, principalement dans son incarnation supérieure, dessert surtout la population anglophone du comté. Il est en grande partie utilisé par elle et, dans une certaine mesure, contre elle. 
Le couple typique de plaideurs n'est donc pas nécessairement composé d'un demandeur anglophone et d'un défendeur acadien. Dans le cas de la cour de circuit, seulement 17 des 73 causes présentées par des demandeurs anglophones locaux sont défendues par des Acadiens, soit $23,3 \%$ du total. En cour de comté, cette proportion est plus élevée, sans être prépondérante, atteignant un peu plus du tiers des causes anglo-brunswickoises. Le niveau d'interaction ethnique est plus élevé dans les deux cours civiles paroissiales. Dans celle de Beresford, toutes les actions intentées par des anglophones du comté, au nombre de 66, l'ont été contre des Acadiens. Des 368 causes présentées par le même groupe dans Saumarez, $316(85,9 \%)$ visaient des Acadiens. Somme toute, la fréquence des causes interethniques est élevée, mais seulement dans les tribunaux inférieurs. Dans les autres, elle est substantielle, mais n'est jamais prépondérante. À première vue, donc, les Acadiens n'apparaissent pas nécessairement les victimes, au niveau supérieur, d'un appareil judiciaire aux mains de seuls demandeurs anglophones. Cette perception est renforcée lorsqu'on examine l'issue des instances. Défendeurs acadiens et anglobrunswickois subissent le même sort: la défaite. Dans la cour de comté, par exemple, le pourcentage de réussite des demandeurs dans les 104 causes dont l'issue est connue et dont les défendeurs sont des Acadiens s'élève à $97,1 \%$, ce qui est identique au pourcentage enregistré pour l'ensemble des causes. Même chose dans les tribunaux inférieurs. Contre les seuls défendeurs acadiens, le pourcentage de réussite est de $99,3 \%$, sur un total de 597 causes, soit le même que pour toutes les causes.

Quant aux demandeurs acadiens, ils entrent surtout en action contre des compatriotes, sauf peut-être en cour de comté. C'est dans ce tribunal que la fréquence des causes entre parties acadiennes est la moins élevée. Elle s'établit néanmoins à 48,3\%, c'est-à-dire que 56 des 116 causes présentées par des demandeurs acadiens sont défendues par d'autres Acadiens. Dans les autres tribunaux, l'homogénéité ethnolinguistique du couple demandeur/défendeur est nettement plus forte. Ainsi, en cour de circuit, 22 des 28 actions acadiennes $(78,6 \%)$ visent d'autres Acadiens. Ce rapport est de 385 sur $422(91,2 \%)$ dans la cour civile paroissiale de Beresford et de 55 sur 64 (85,9\%) dans celle de Saumarez. Qui plus est, peu importe le tribunal, les demandeurs acadiens ne sont pas défavorisés par rapport aux demandeurs anglophones. Comme les autres, ils sortent victorieux des démarches judiciaires dans la quasi-totalité des $\operatorname{cas}^{46}$. Compte tenu de la fré-

46 En cour de circuit, par exemple, les demandeurs acadiens obtiennent gain de cause dans 11 cas sur $12(91,7 \%)$, alors que les autres demandeurs réussissent dans 39 cas sur 44 $(88,6 \%)$. L'écart est plus prononcé en cour de comté, sans toutefois être significatif. Au total, 
quence des causes intra-ethniques, il semble donc que les demandeurs acadiens n'aient pas eu trop de réticences à chercher devant l'appareil judiciaire néo-brunswickois le règlement de litiges avec des compatriotes. Ce constat ouvre une autre brèche dans l'hypothèse d'un évitement généralisé des tribunaux au sein de la communauté acadienne à la fin du XIX ${ }^{\mathrm{e}}$ siècle. Certes, les Acadiens ont proportionnellement moins recours aux tribunaux que les anglophones du comté, mais quand ils y ont recours, c'est souvent pour régler des litiges avec des compatriotes. S'il y avait eu boycottage généralisé et systématique, on peut supposer que c'est tout particulièrement dans la catégorie des litiges intra-ethniques qu'il se serait manifesté. Il y a donc recours judiciaire acadien dans les tribunaux civils de Gloucester. Un élément le distingue toutefois jusqu'à présent de celui des AngloBrunswickois: son ampleur. Reste à savoir s'il se distingue également sur le plan du contenu.

\section{3 - LA NATURE DES CAUSES}

Des trois grandes sphères d'activité judiciaire des tribunaux civils, les questions pécuniaires ou dettes, la propriété (mobilière et immobilière) et les personnes ${ }^{47}$, une seule rassemble la plupart des interventions judiciaires des Acadiens à la fin du siècle: celle des questions pécuniaires (voir tableau 3). Elle prédomine nettement dans deux types de tribunaux sur trois. D'abord dans la cour de comté, où plus des deux tiers des 48 actions acadiennes pour lesquelles la nature du litige est connue portent sur le recouvrement d'une somme due. Puis dans les tribunaux inférieurs, où presque toutes les actions présentées par des Acadiens, soit 466 sur 474 (98,3\%), portent sur une question pécuniaire. Dans ces deux cours, le comportement judiciaire acadien présente le même profil - caractérisé par la prédominance des litiges pécuniaires - que celui des autres plaideurs du comté, tout en étant moins accentué, particulièrement en cour de comté. Ce type

26 causes acadiennes sur $30(86,7 \%)$ et 134 causes anglophones sur $140(95,7 \%)$ se terminent par la victoire du demandeur. Cette proportion est pratiquement la même dans les tribunaux inférieurs, s'établissant à 305 sur $310(98,4 \%)$ dans le premier cas et à 413 sur $414(99,8 \%)$ dans le second. On remarque la même tendance dans d'autres ressorts. Voir Wayne V. McIntosh, "A State Court's Clientele: Exploring the Strategy of Trial Litigation», Law and Society Review, 19,3 (1985): 435-441.

47 Ce classement reprend en le modifiant quelque peu le classement traditionnel établi en fonction de la cause d'action. Il a été conçu pour refléter les préoccupations de l'historien. Il est basé sur les éléments impliqués dans l'incident ou le conflit donnant lieu à une action. Ainsi, une action en responsabilité délictuelle découlant de la destruction d'une partie d'une récolte a été classée sous la catégorie «propriété» plutôt que sous la cause d'action «délit civil». 
TABLEAU 3

Nature des actions dans les tribunaux civils de Gloucester, de 1873 à 1899

\begin{tabular}{|c|c|c|c|}
\hline Nature & $\begin{array}{c}\mathbf{A} \\
\text { Nbre (\%) }\end{array}$ & $\begin{array}{c}\mathbf{B} \\
\text { Nbre (\%) }\end{array}$ & $\begin{array}{c}\mathbf{T} \\
\text { Nbre (\%) }\end{array}$ \\
\hline \multicolumn{4}{|l|}{ Circuit } \\
\hline Dettes & $4(14,8)$ & $44(67,7)$ & $79(54,5)$ \\
\hline Propriété & $17(63,0)$ & $19(29,2)$ & $58(40,0)$ \\
\hline Personnes & $6(22,2)$ & $2(3,1)$ & $8 \quad(5,5)$ \\
\hline Divers & $0 \quad(0,0)$ & $0 \quad(0,0)$ & $0 \quad(0,0)$ \\
\hline s.t. & 27 & 65 & 145 \\
\hline Indéterminé & 1 & 8 & 17 \\
\hline Total & 28 & 73 & 162 \\
\hline \multicolumn{4}{|l|}{ Comté } \\
\hline Dettes & $33(68,8)$ & $177(95,2)$ & $375(92,1)$ \\
\hline Propriété & $11(22,9)$ & $5 \quad(2,7)$ & $18 \quad(4,4)$ \\
\hline Personnes & $3(6,3)$ & $1 \quad(0,5)$ & $4 \quad(1,0)$ \\
\hline Divers & $1 \quad(2,1)$ & $3(1,6)$ & $10 \quad(2,5)$ \\
\hline s.t. & 48 & 186 & 407 \\
\hline Indéterminé & 68 & 160 & 390 \\
\hline Total & 116 & 346 & 797 \\
\hline \multicolumn{4}{|l|}{ Inférieurs } \\
\hline Dettes & $466(98,3)$ & $424(99,3)$ & $942(98,8)$ \\
\hline Propriété & $5 \quad(1,1)$ & $0 \quad(0,0)$ & $5 \quad(0,5)$ \\
\hline Personnes & $0 \quad(0,0)$ & $0 \quad(0,0)$ & $0 \quad(0,0)$ \\
\hline Divers & $3(0,6)$ & $3(0,7)$ & $6 \quad(0,6)$ \\
\hline s.t. & 474 & 427 & 953 \\
\hline Indéterminé & 12 & 7 & 20 \\
\hline Total & 486 & 434 & 973 \\
\hline
\end{tabular}

Source: Voir note 14.

A: Acadiens, B: ANglo-Brunswickois de Gloucester, T: Total

d'action compose en effet $95,2 \%$ de l'activité judiciaire des autres plaideurs locaux en cour de comté, sur un total de 186 actions documentées. De même, il représente 99,3\% des 427 actions documentées du groupe dans les cours inférieures. La situation est toutefois différente pour les Acadiens dans le principal tribunal du comté, la cour de circuit. Les matières d'endettement y occupent une place beaucoup moins importante: seulement quatre causes acadiennes, sur un total de $27(14,8 \%)$, ont pour objet le recouvrement de sommes dues. Ce n'est pas le cas des causes présentées par les autres citoyens du comté, qui sont encore largement dominées par des litiges pécuniaires. On en compte 44 sur un total de 65 , soit $67,7 \%$ des causes documentées. 
Ce sont surtout les litiges entourant la perception de comptes qui sont les plus fréquents dans la sphère pécuniaire. C'est pour un tel motif que Jacques P. Doucet se présente, par exemple, devant le magistrat de Beresford en 1891 pour réclamer d'Antoine Grant la somme de $4 \$$, en guise de paiement pour cinq barils de pommes de terre livrés en $1889^{48}$. Un autre type de litige est presque aussi fréquent en cour de circuit et en cour de comté: les actions sur des effets de commerce négociables, généralement des billets à ordre. C'est le cas, par exemple, de l'action introduite par Ubalde Landry contre Patrick Foley en cour de comté en 1878, pour forcer ce dernier à honorer un billet à ordre. Foley s'était engagé deux ans plus tôt à lui payer «la somme de $\$ 9.00$ piastres argent courant [avant] le promies D'aluit prochain (sic)» ${ }^{49}$. Quant aux autres types de litiges regroupés sous la catégorie des dettes, ils sont peu fréquents. Il s'agit surtout d'actions portant sur des questions de loyer, de salaire ou d'honoraires.

Après les questions pécuniaires, les questions de propriété sont le plus souvent au centre des interventions judiciaires des Acadiens de Gloucester. De tels litiges occupent la première place dans la cour de circuit et la deuxième dans la cour de comté ainsi que dans les tribunaux inférieurs. Dans la cour de circuit, plus de la moitié des actions initiées par des demandeurs acadiens visent à régler un litige sur la propriété. Cette sorte de litige est moins présente dans les deux autres cours. Elle est à l'origine de $22,9 \%$ des actions dans la cour de comté et de $1,1 \%$ dans les tribunaux inférieurs. Tout comme en matière de dettes, les recours judiciaires acadien et anglobrunswickois diffèrent non pas tant sur le plan de l'orientation générale, mais plutôt sur celui de l'amplitude. Dans les deux cas, la catégorie des litiges sur la propriété vient au deuxième rang. À la différence des Acadiens, toutefois, les Anglo-Brunswickois de Gloucester présentent proportionnellement moins d'actions en matière de propriété dans les trois types de tribunaux, soit $29,2 \%$ en cour de circuit, $2,7 \%$ en cour de comté et $0,0 \%$ dans les tribunaux inférieurs. Du côté des Acadiens, un type particulier de litige occupe une position prépondérante dans chaque tribunal, reflétant ainsi partiellement le partage des compétences. En cour de circuit, les actions en matière de contestation de titres de propriété, comme celle intentée par John M. Haché de Caraquet en 1894 contre un autre habitant de la paroisse, Gervais Godin ${ }^{50}$, viennent au premier rang. En cour de comté, par

La catégorie «personnes» réunit les litiges dans lesquels des personnes sont moralement ou physiquement impliquées: voies de fait, atteinte à la réputation, bris de promesse de mariage, arrestation illégale, poursuite malveillante. Voir Couturier, La justice civile..., 178-179.

48 APNB, RG 8, RS 495, A/2-a, Parish of Beresford, 29-30, Doucet c. Grant, 1891.

49 APNB, RG 6, RS 432, A, boîte 1878-1880, Landry c. Foley, 1878.

50 APNB, RG 5, RS 42, 1894, z(2), Haché c. Godin, 1894. 
contre, la quasi-totalité des causes en matière de propriété, moins nombreuses que dans le premier tribunal, portent sur l'appropriation illicite de biens. Cette attribution est également la plus populaire dans les tribunaux inférieurs, même si elle est rarement exercée.

Finalement, les Acadiens ont très rarement recours aux tribunaux pour régler des litiges concernant des relations entre personnes. Peu d'individus imitent le geste de Dominique Gallien qui, en 1894, a recours aux services du juge de la cour de circuit pour obtenir réparation après avoir été agressé physiquement ${ }^{51}$. Cette catégorie de litiges ne regroupe en effet que six actions dans la cour de circuit, trois dans la cour de comté et aucune dans les deux cours civiles paroissiales. L'activité judiciaire acadienne dans la catégorie est saupoudrée parmi divers types de litiges: voies de fait, arrestation illégale, atteinte à la réputation, poursuite malveillante. Ce n'est pourtant pas parce qu'ils sont Acadiens qu'ils évitent les tribunaux pour ce genre de litige: les Anglo-Brunswickois semblent y avoir encore moins recours. Ils n'initient que deux actions dans le premier tribunal, une dans le second et aucune dans le dernier. Tout le champ des relations sociales est donc peu couvert par les tribunaux civils de Gloucester à la fin du $\mathrm{XIX}^{\mathrm{e}}$ siècle.

L'activité judiciaire acadienne présente donc des particularités sur le plan de la nature des causes. Elle est plus dispersée dans l'ensemble des champs d'activité, moins concentrée dans le domaine du recouvrement de créances, particulièrement en cour de circuit. Or c'est surtout dans ce domaine que les tribunaux civils sont actifs au $\mathrm{XIX}^{\mathrm{e}}$ siècle. Ils constituent essentiellement des instruments de régulation du système de crédit commercial et de crédit à la consommation $^{52}$. À preuve, dans la cour de circuit, plus de la moitié des actions peuvent être classées dans la catégorie des litiges pécuniaires. Cette proportion grimpe à $92 \%$ dans la cour de comté et à près de $100 \%$ dans les tribunaux inférieurs. Or les Acadiens présentent proportionnellement moins d'actions pécuniaires que les autres habitants de Gloucester. C'est donc la faiblesse de l'activité judiciaire acadienne dans le domaine des litiges pécuniaires qui rend le mieux compte de la faiblesse générale de l'activité judiciaire du groupe, tant en cour de circuit qu'en cour de comté. D'ailleurs, dans les deux autres domaines,

51 Ibid., Gallien c. Frigault, 1894.

52 Cet aspect a été démontré dans nombre d'études. Voir, entre autres, pour les tribunaux supérieurs: Kolish, «Some Aspects...», 337-365, et Clinton W. Francis, «Practice, Strategy, and Institution: Debt Collection in the English Common-Law Courts, 1740-1840», Northwestern University Law Review, 80 (hiver 1986): 807-955. Pour le niveau inférieur, voir Sylvio Normand, «Justice civile et communauté rurale au Québec, 1880-1920», Les cahiers de Droit, 25,3 (septembre 1984): 579-615. 
la propriété et les personnes, les Acadiens sont aussi sinon plus actifs que les autres plaideurs du comté. Ainsi, dans la cour de circuit, ils présentent au total sensiblement le même nombre d'actions que les Anglo-Brunswickois. Pour les seules neuf dernières années, ils en présentent toutefois 11 tandis que les autres en présentent six. En cour de comté, le scénario est le même pour la période 1873-1890. Quatorze actions sont présentées par des Acadiens et six par des Anglo-Brunswickois. Ces données, même si elles sont limitées, montrent assez bien que dans les domaines non pécuniaires, la contribution acadienne à l'activité judiciaire tend à mieux refléter la position démographique du groupe. Or ces domaines ne recouvrent pas des types de litiges nécessitant obligatoirement une intervention coercitive des tribunaux. Plusieurs peuvent très bien être réglés hors cours, ce qui n'est peut-être pas le cas d'un litige pécuniaire, quand vient le temps, par exemple, d'obtenir le remboursement d'une dette. C'est donc dire qu'en des matières où le frein ethnolinguistique ou culturel pourrait être le plus fort, les Acadiens ont néanmoins recours aux cours de justice, tout aussi souvent et parfois plus encore que les anglophones. Il s'agit là d'indices supplémentaires de la fragilité de l'hypothèse d'un évitement généralisé des tribunaux. Ce qu'il reste à expliquer, c'est pourquoi ils n'y ont pas plus souvent recours en matière de dettes. Il faut pour cela se tourner du côté du profil des plaideurs.

\section{4 - PROFIL ÉCONOMIQUE DES PLAIDEURS}

Deux grands types de plaideurs, provenant de divers domaines d'activité, interviennent devant les tribunaux civils de Gloucester durant les dernières décennies du XIX ${ }^{\mathrm{e}}$ siècle: des plaideurs apparemment libres de tout lien commercial ou administratif, simples pêcheurs, fermiers, ou artisans, et des plaideurs du secteur des affaires, marchands, représentants, entreprises ou institutions financières ${ }^{53}$. Ce sont les premiers, de simples particuliers, qui sont les principaux animateurs de l'activité judiciaire acadienne. La situation est on ne peut plus claire en cour de circuit. Près des trois quarts des causes dont le profil de la partie demanderesse est connu sont présentées par des particuliers (voir tableau 4). Du côté anglo-brunswickois, par contre, ce type de plaideur ne présente que le quart des causes. On retrouve le même phénomène en cour de comté. Sur 95 causes acadiennes, environ la moitié sont introduites par de simples particuliers. Or seule-

53 Sur le classement, voir Couturier, La justice civile..., 332-334. Voir également McIntosh, «A State Court's...», 421-448. 
TABLEAU 4

Profil économique des demandeurs dans les tribunaux civils de Gloucester, de 1873 à 1899

\begin{tabular}{lrrr}
\hline Tribunal/Profil & \multicolumn{1}{c}{ A } & B & T \\
& Nbre (\%) & Nbre (\%) & \\
\hline Circuit & & & \\
Particuliers & $17(73,9)$ & $15(25,9)$ & 32 \\
Gens d'affaires/entreprises & $6(26,1)$ & $37(63,8)$ & 57 \\
Autres & $0(0,0)$ & $6(10,3)$ & 36 \\
$\quad$ s.t. & 23 & 58 & 125 \\
Indéterminé & 5 & 15 & 37 \\
Total & $\mathbf{2 8}$ & $\mathbf{7 3}$ & $\mathbf{1 6 2}$ \\
Comté & & & \\
Particuliers & $47(49,5)$ & $58(19,9)$ & 110 \\
Gens d'affaires/entreprises & $40(42,1)$ & $206(70,5)$ & 396 \\
Autres & $8(8,4)$ & $28(9,6)$ & 118 \\
$\quad$ s.t. & 95 & 292 & 624 \\
Indéterminé & 21 & 54 & 173 \\
Total & $\mathbf{1 1 6}$ & $\mathbf{3 4 6}$ & $\mathbf{7 9 7}$ \\
Inférieurs & & & \\
Particuliers & & 14 & 238 \\
Gens d'affaires/entreprises & 199 & 413 & 643 \\
Autres & & & 17 \\
$\quad$ s.t. & & & 75 \\
Indéterminé & & & $\mathbf{9 7 3}$ \\
Total & & &
\end{tabular}

Source: Voir note 14.

A:Acadiens, B: Anglo-Brunswickois, T: Total

ment $19,9 \%$ des 292 causes introduites par d'autres plaideurs locaux le sont par des membres du même groupe. Si la présence de particuliers est moins prononcée dans les causes acadiennes en cour de comté, elle n'en continue pas moins de se démarquer nettement du profil des causes des autres plaideurs du comté. Finalement, dans les cours locales, les simples particuliers assument toujours la plus grande partie de l'activité judiciaire acadienne, contrairement à la situation qui prévaut chez les autres plaideurs du comté. Environ la moitié des causes acadiennes sont introduites par ce type de plaideur, contre moins de 5\% dans celles du second groupe. C'est donc dire que, parmi l'ensemble des plaideurs acadiens, la plus grande place n'est pas occupée par des marchands et des gens d'affaires, mais par de simples particuliers, agissant à titre personnel. Ces gens ont recours aux 
tribunaux civils pour régler des litiges qui, ont-ils jugé, ne pouvaient pas être réglés autrement ou mieux réglés ailleurs. Donc, le recours judiciaire acadien n'est pas assumé par la seule élite économique, si petite soit-elle, mais s'étend également à d'autres groupes, ce qui n'est pas le cas du côté des Anglo-Brunswickois. Et même s'il est probablement plus facile pour de simples particuliers de choisir des mécanismes informels, tels la médiation ou l'arbitrage d'un notable ou du curé, pour régler un litige, plusieurs optent néanmoins pour les mécanismes officiels.

Par ailleurs, les gens d'affaires acadiens semblent également proportionnellement moins actifs que les gens d'affaires anglophones, principalement dans les deux tribunaux supérieurs. Sur les 43 actions attribuables à des gens d'affaires locaux dans la cour de circuit entre 1873 et 1899 , seulement $14 \%$ proviennent d'Acadiens. Une situation similaire prévaut également dans la cour de comté, où environ $16 \%$ des actions introduites par des gens d'affaires l'ont été par des Acadiens. Cette situation reflète en partie la structure des interventions acadiennes. Plusieurs des plaideurs anglophones du milieu des affaires sont des plaideurs répétitifs ${ }^{54}$. Kennedy F. Burns, commerçant et entrepreneur forestier, présente probablement le cas le plus probant. Il initie 13 actions en cour de circuit et 46 en cour de comté, soit en son nom propre, soit à travers ses diverses sociétés commerciales. On ne rencontre pas le même phénomène chez les Acadiens, sauf dans la cour civile paroissiale de Beresford. Ainsi, aucun Acadien ne figure parmi les plaideurs ayant présenté plus de dix actions en cour de circuit ou en cour de comté. De même, dans la cour de Saumarez, six des huit principaux plaideurs sont anglo-brunswickois. C'est toutefois un homme d'affaires acadien qui est le principal plaideur dans l'autre cour civile paroissiale. Frédérick Comeau y présente en effet 74 actions, soit près d'une cinquantaine de plus qu'un autre commerçant, Joseph Windsor. Or l'Acadien Comeau et les autres principaux plaideurs, des anglophones, semblent partager une même caractéristique. Tous sont de grands acteurs dans le domaine économique, chacun dans un secteur d'activité ou dans une zone d'influence ${ }^{55}$. Il semble donc exister une certaine coïncidence entre la taille et le niveau d'activité. Or les quelques éléments disponibles sur le monde des affaires acadien permettent de supposer que la plupart des intervenants sont de petite taille, expliquant ainsi en partie le plus faible recours judiciaire des gens d'affaires du groupe.

54 Couturier, La justice civile..., 412-433.

55 Ibid. 
Un autre élément, l'évolution de l'activité judiciaire des gens d'affaires dans le temps, jette également un éclairage différent sur la contribution des gens d'affaires acadiens ${ }^{56}$. Celle-ci change vraisemblablement au cours de la période ${ }^{57}$. Largement inférieure à celle des Anglo-Brunswickois durant les années 1873-1881, elle atteint progressivement un niveau similaire à celui de la présence du groupe dans la communauté des affaires au cours des années 1882-1890 et 1891-1899, tant en cour de circuit qu'en cour de comté. Ainsi, durant la première période, aucune cause n'est acadienne en cour de circuit et seulement 16 sur $160(10 \%)$ le sont en cour de comté. Or selon les données des annuaires de crédit de la firme Dun, Wiman \& Co., un peu moins du sixième des gens d'affaires sont acadiens en $1873^{58}$. Au cours de la période suivante, le pourcentage d'actions acadiennes est plus élevé, s'établissant à $14,3 \%$ dans la cour de circuit et à $17,3 \%$ dans la cour de comté. De même, la proportion de gens d'affaires acadiens semble progresser; elle passe de $16,2 \%$ en 1882 à $23,7 \%$ en $1887^{59}$. Finalement, durant la dernière période, qui couvre les années 1891-1899, cinq causes sur $12(41,6 \%)$ peuvent être considérées acadiennes dans le premier tribunal et 15 sur $34(44,1 \%)$ dans le second. Ces chiffres reflètent la position des Acadiens parmi la communauté des affaires. Ceux-ci forment environ 33,9\% du groupe en 1890, $46,3 \%$ en 1895 et $43,5 \%$ en $1899^{60}$. Même si ces quelques résultats ne doivent pas être perçus comme dépeignant avec exactitude le niveau réel de l'activité judiciaire de chaque groupe, ils semblent toutefois exprimer assez clairement une chose: il n'y a peut-être pas de différence profonde ou même significative dans les deux tribunaux supérieurs entre l'activité judiciaire des gens d'affaires acadiens et celle des autres durant les deux dernières décennies du siècle. Du moins apparaissent-elles largement atténuées. C'est donc dire que la

56 Une telle analyse, quoique nécessaire en raison de la variation de l'activité judiciaire acadienne, présente des difficultés. Par période, l'échantillon disponible est très faible, ce qui a pour effet d'accroître l'impact des aléas de l'identification et du classement des causes. C'est pourquoi les données doivent être interprétées avec prudence.

57 Cette notion de changement est importante. Elle ne peut toutefois pas être mesurée avec précision sur une période aussi courte et avec des données incomplètes. Elle est suggérée notamment par l'analyse de l'activité judiciaire acadienne dans deux domaines, la propriété et les personnes. Là aussi, l'activité judiciaire acadienne atteint dans l'avant-dernière décennie un niveau reflétant le poids des Acadiens dans la démographie du comté. Elle l'est également par l'évolution du niveau général d'activité judiciaire acadienne dans la cour de circuit et l'évolution du taux de litiges pour mille habitants dans les deux tribunaux supérieurs. Tous ces indices, certes fragiles, mais nombreux, semblent indiquer que les choses changent au fil des années 1870.

58 The Mercantile Agency..., juillet 1873.

59 Ibid., juillet 1882; Ibid., juillet 1887.

60 Ibid., juillet 1890; Ibid., juillet 1895; Ibid., juillet 1899. 
sous-utilisation acadienne des tribunaux civils néo-brunswickois est peut-être en grande partie le reflet de la sous-représentation acadienne parmi le groupe des grands utilisateurs de l'appareil judiciaire, à savoir les gens d'affaires ${ }^{61}$. C'est là un élément majeur de la compréhension de l'activité judiciaire acadienne.

Jusqu'à présent, les historiens de l'Acadie ont principalement envisagé la question de la régulation juridique en termes ethnoculturels. Ces dernières données, tout comme celles sur la nature des causes, montrent qu'il faut également l'envisager en termes socioéconomiques. C'est en effet probablement la situation économique de la société acadienne qui rend le plus directement compte de l'ampleur et de la nature de l'activité judiciaire acadienne. D'abord la sousreprésentation acadienne parmi la communauté des affaires, ainsi que la forme même de sa présence. Puis le niveau de vie des Acadiens de Gloucester. Et finalement le sous-développement économique du groupe et de la région. Certes les Acadiens du comté de Gloucester paraissent n'avoir «point de fort pour la loi» à la fin du XIX ${ }^{\mathrm{e}}$ siècle; ils ont moins recours aux tribunaux civils que les Anglo-Brunswickois durant la période examinée. Mais ils ne les boycottent pas systématiquement pour autant. Il y a dans la société acadienne une certaine connaissance du droit et des recours en droit. Et il y a utilisation des tribunaux. Si l'activité judiciaire des Acadiens est moindre, c'est en grande partie parce qu'ils n'ont pas trop besoin de se servir de cet instrument spécialisé, étant donné que peu d'entre eux sont touchés par le domaine principal d'intervention des tribunaux civils, la régulation des activités commerciales et financières. Pour ce qui est des autres matières, notamment celles relevant des relations sociales, tout comme les autres habitants de Gloucester, ils les règlent généralement hors des tribunaux civils. Mais quand ils ont besoin de se servir des cours de justice, ils s'en servent, souvent même contre des compatriotes, ce qui renforce également la perception que le facteur ethnoculturel n'est pas au premier plan. Incarné surtout par l'unilinguisme de l'appareil judiciaire et la sous-représentation acadienne parmi les officiers et auxiliaires de justice, celui-ci joue, mais seulement de manière limitée. Pour la plupart des plaideurs potentiels acadiens de Gloucester, le recours aux tribunaux civils constitue donc une solution parmi d'autres pour régler des conflits. Ce n'est pas dire que les autres mécanismes de gestion des conflits, notamment les mécanismes informels, ne jouent aucun rôle; c'est simplement dire que les mécanismes étatiques jouent aussi un rôle, en grande partie déterminé par les besoins auxquels ils répondent habituellement en

61 Pour une interprétation similaire, voir Kolish, «Some Aspects...», 348-351. 
cette fin de siècle. Trop accaparée par les premiers, l'historiographie a négligé de montrer l'importance des seconds. C'est ce que nous avons cherché à faire.

Certaines questions restent toutefois en plan. Il en est ainsi de l'évolution dans le temps du comportement judiciaire acadien. À première vue, il ne semble pas changer radicalement entre 1873 et 1899 , même si des signes de changement existent, particulièrement dans les années 1870. Il faudrait cependant l'étudier sur une plus longue période, en particulier en amont, durant les dernières décennies du $\mathrm{XVIII}^{\mathrm{e}}$ siècle et les premières du $\mathrm{XIX}^{\mathrm{e}}$ siècle, pour tenter d'en mettre au jour les racines ${ }^{62}$. Ce qui est clair, c'est que la transformation de la culture juridique acadienne n'est ni amorcée, ni complétée, durant la période 1873-1899. Les différences régionales devraient également être prises en charge. Gloucester est peut-être, parmi les comtés à forte population acadienne, le comté où le niveau d'activité judiciaire acadienne est le plus faible, les conditions locales y étant peu propices. D'autres comtés, Westmorland ou Madawaska, par exemple, pouvaient compter sur un rapport avocat acadien/population acadienne plus favorable ou sur une économie plus engagée dans la voie de la modernisation. Le recours judiciaire acadien s'en ressent-il? Par ailleurs, outre la dimension civile, le volet criminel de l'activité judiciaire devrait aussi être pris en charge. Finalement, justice informelle et justice officielle ont été jusqu'ici examinées séparément. Il faudrait maintenant chercher à comprendre l'interaction entre les deux, voir comment s'opère de manière globale la régulation juridique dans la société acadienne.

Longtemps l'image dominante de la société acadienne dans l'historiographie a été celle d'une société unidimensionnelle, définie presque exclusivement par la dimension nationale, à travers notamment l'examen du discours des élites clérico-laïques. C'est une autre vision de la société acadienne des dernières décennies du XIX ${ }^{\mathrm{e}}$ siècle qui est mise en lumière par l'examen des archives judiciaires des tribunaux civils de Gloucester. Elle s'y révèle plus complexe, pluridimensionnelle. Plusieurs éléments l'attestent. D'abord la simple présence au sein de la communauté de conflits, de disputes, c'est-àdire des éléments longtemps négligés par une historiographie souvent engagée dans la recherche d'un consensus nationalitaire. Puis le degré de pluralisme juridique qui semble régner dans la communauté. Et finalement le fait que les litiges ne sont pas traités seulement dans un

62 Voir Marie-Claire Pitre, «Les Acadiens...», 184, qui tente à partir de données limitées de faire remonter aux années 1827-1848 les «premiers balbutiements de l'intégration de la société acadienne sur la scène socio-juridique britannique». 
cadre communautaire, ce qui est le propre des sociétés traditionnelles, mais également par des mécanismes en partie extérieurs à la communauté, mis sur pied par l'État. Ce dernier aspect est lourd de signification. Il semble impliquer que dans le domaine de la justice civile, secteur certes de second plan parmi les grands secteurs de la vie collective, mais néanmoins vital pour le gouvernement de la société, surtout en matière économique, il y a intégration subtile mais bien réelle des Acadiens à la société globale néo-brunswickoise à la fin du $\mathrm{XIX}^{\mathrm{e}}$ siècle. Certes, l'intégration n'est pas complète, mais elle est plus importante que ce à quoi on pouvait s'attendre en s'appuyant sur le seul discours face aux tribunaux. Société partiellement intégrée, société complexe: voilà deux concepts explicatifs qu'un examen de l'activité judiciaire a permis de mettre au jour et qu'il faudrait examiner plus à fond pour mieux comprendre, puis mieux décrire l'expérience historique acadienne des dernières décennies du XIX ${ }^{\mathrm{e}}$ siècle. 